\title{
Relationship between three intensity levels of Toxocara canis larvae in the brain and effects on exploration, anxiety, learning and memory in the murine host
}

\author{
D.M. Cox and C.V. Holland* \\ Department of Zoology, Trinity College, Dublin 2, Ireland
}

\begin{abstract}
Outbred LACA mice were administered low (100 ova), medium (1000 ova), high (3000 ova) and trickle $(4 \times 250$ ova) doses of Toxocara canis ova and the effect of infection was examined with respect to the number of larvae recovered from the brain and their behaviour. Recovery of larvae from the brain was generally low with the \% recovery expressed in terms of the total dose administered being highest for the 3000 dose $(6.1 \%)$ and 1000 dose $(6 \%)$, followed by the $100(4.4 \%)$ and trickle (3.5\%) doses. The variation in larval recoveries was large between individual mice receiving similar doses. The level of infection in the brain was lower in mice receiving a multiple as opposed to an equivalent single dose of ova. Mice were then divided into three larval intensity groupings based upon the number of larvae recovered from their brain. The ranges for the groups were as follows: low intensity group, 0-15 larvae; moderate intensity group, 27-55 larvae; high intensity group, 66-557 larvae. Three behavioural tests were carried out on control and infected mice. Exploration and response to novelty was examined using a ' $\mathrm{T}$ ' maze and learning was investigated by means of a water-finding task. Anxiety was measured using an elevated plus maze apparatus. Infected mice were less explorative and less responsive to novelty in the ' $T$ ' maze and this was particularly pronounced for the heavily infected mice. In the elevated plus maze, infected mice displayed reduced levels of anxiety to aversive and exposed areas of the maze, particularly in the case of the moderate and high intensity mice. There was evidence for impaired learning ability in the water task apparatus for moderate and high intensity mice. In general, the effects of infection on behaviour were more pronounced in the moderate and high intensity groups compared to the low intensity group.
\end{abstract}

\section{Introduction}

Toxocara canis is a nematode which infects canines worldwide and, as a consequence of the widespread environmental dissemination of its ova in host faeces, other abnormal hosts, including humans, are exposed to the parasite. Upon entry into an abnormal host, the immature stage of $T$. canis undergoes a somatic migration through the organs of the body but fail to reach maturity

*Fax: +35316778094

E-mail: cholland@mail.tcd.ie as adult worms in the intestine. Exposure to Toxocara, as measured indirectly by serology, is widespread in humans, for example 31\% of Irish schoolchildren were found to exhibit positive serology at a cut-off titre of 1:50 (Holland et al., 1995). Despite this, the relationship between Toxocara infection and morbidity requires further investigation and the public health significance of toxocariasis, other than ocular disease, is still the subject of some controversy (Holland, 1997).

Toxocara is known to produce three recognized clinical entities, i.e. visceral larva migrans (Beaver et al., 
1952), ocular larva migrans (Shields, 1984) and covert toxocariasis (Taylor et al., 1988). In addition to these recognized syndromes, there is also evidence to suggest that the parasite may have the potential to alter the behaviour of the host, due to the neurotrophic behaviour of the larvae, which results in a greater concentration of parasite in the brain as the infection progresses (Sprent, 1955; Dunsmore et al., 1983; Skerrett \& Holland, 1997). Several studies, based upon a single dose of T. canis ova, reported larvae in a non-random distribution within the brain (Burren, 1971; Dolinsky et al., 1981; Summers et al., 1983). Larvae were recorded from the heavily myelinated tracts of the corpus callosum, internal and external fornix capsules, cerebellar peduncles and the cerebellar medulla (Summers et al., 1983).

Toxocara infection in the mouse is a useful hostparasite system for two reasons. Firstly, it can act as a model for human infection and observed changes in murine behaviour, particularly at low dose levels, may be relevant to humans with toxocariasis (Holland, 1997). Secondly, it can test the hypothesis that parasite-altered host behaviour may contribute to increased predation of infected paratenic hosts and hence enhanced transmission of the parasite (Moore \& Gotelli, 1990). In a previous paper, Cox \& Holland (1998) described the relationship between the numbers of larvae recovered from the brain of $T$. canis-infected mice (as opposed to the dose administered) and social behaviour and anxiety (measured using the light/dark box and novel odours).

In those experiments, the mice received a single dose of 2000 ova which produced significant variation in larval burdens between individual mice and resulted in two intensity groupings described as low (less than 50 or 70 larvae per brain depending upon the experiment) or high (greater than 50 or 70 larvae per brain depending upon the experiment). In the experiments described in this paper, two novel elements are included - firstly groups of mice received one of four doses - low, trickle, medium and high doses which resulted in the creation of three intensity groupings, the low and high group being very different to those which resulted from a single dose of 2000 ova. Secondly, the effect of infection upon different behaviours such as learning and memory are described and the test for response to exploration, novelty and anxiety uses different paradigms - the ' $\mathrm{T}$ ' maze and the elevated plus maze.

\section{Materials and methods}

\section{Mouse maintenance}

Forty-five outbred (LACA) mice, aged between 4 and 5 weeks were used in the experiment. The mice were housed in an animal maintenance room in single cages measuring $35 \times 15 \times 13 \mathrm{~cm}$. The room was illuminated by a 200 lux bulb, which operated on a $12 \mathrm{~h}$ daily cycle $(0800$ lights on - 2000 lights off). The room was kept at a constant temperature of $22^{\circ} \mathrm{C} \pm 2{ }^{\circ} \mathrm{C}$ and humidity of $50 \pm$ $10 \% \mathrm{RH}$ which was monitored daily. An automatic fan circulated a continuous stream of warm air around the room. The mice were fed Redmills Commercial Rodent Nuts and free access to food and water was available at all times. The mice were weighed individually and grouped according to their weight, placing similar weights together (range 26.1-43.2 g). Each mouse was then assigned to one of five groups. For identification purposes, each group was assigned a number. The mice were habituated to these conditions for 2 weeks before infection or behavioural tests were carried out.

\section{Infection of mice}

The doses of T. canis ova administered were 100 (low dose), 1000 (medium dose), 1000 over 28 days, i.e. 250 per week (trickle) and 3000 (high). Four of the groups of mice were randomly chosen and infected orally by stomach intubation with one of the four infection doses suspended in $0.2 \mathrm{ml}$ of distilled water. The control group was sham-inoculated with distilled water. The mice were monitored hourly after infection for post-inocula trauma or ill effects. The infection was allowed to establish over a 30-day period before behavioural testing began. Previous research had demonstrated that in mice infected with $T$. canis ova the larvae reach the brain within 2 days and stabilize at this site between days 35 and 45 (Burren, 1971). It was considered that the behavioural effects due to the presence of the larvae in the brain would be most conspicuous during this time.

\section{Behavioural testing}

Behavioural assessment was carried out in the room were the mice were housed. The ' $\mathrm{T}$ ' maze, elevated maze and water task experiments were run on consecutive days and testing was carried out between 1000 and 1800 h. Each individual experiment was recorded on videotape using a Sony Camcorder Recorder attached to a video monitor which allowed the animal's behaviour to be observed from a position in which the animal could not see the experimenter. After each mouse had been tested, faecal pellets were removed and the whole apparatus swabbed with dilute alcohol solution before the next mouse was exposed to the apparatus. The data were collected and recorded by analysing the prerecorded videos in which the infection status of each group was unknown.

\section{Behavioural tests}

The investigation of exploratory behaviour and response to novelty using the ' $T$ ' maze paradigm

The ' $\mathrm{T}$ ' maze paradigm was used to measure several parameters such as general locomotion, areas visited, rearing and investigation of a novel object placed within the maze. The reaction of the mice to an unfamiliar environment was assessed over 2 days and then after habituation. The running wheel was introduced into the left arm while the right arm and start alley remained familiar.

The test apparatus is a ' $T$ ' maze made of plywood and coated with varnish. The maze has a start box and two choice arms. A guillotine door separated the start box from an alley leading to the choice point. At the end of each choice arm was an end box which was situated in such a way that the mouse had to turn $90^{\circ}$ to enter. The maze was covered with opaque plexiglass in order to 
prevent the mice from escaping. The end boxes were left open-topped to accommodate the novel running wheel.

Testing was proceeded by 2 days of habituation to the apparatus. During habituation, each mouse was allowed free access to the maze for a period of $5 \mathrm{~min}$ per day during which the following activities were recorded: the number of times each mouse entered the right arm, left arm and start alley of the maze, and the number of rears performed per mouse. A rear was defined as the front paws being lifted off the floor and placed on the side of the apparatus or being held close to the body (Cox, 1996).

The mice were then tested for 1 day. Testing began by placing the mouse in the start box for $30 \mathrm{~s}$. After this time had elapsed, the guillotine door was raised, the animal's activity monitored over a $5 \mathrm{~min}$ period and the following activities were recorded: the number of times each mouse entered the right arm, left arm and start alley of the maze; the number of rears performed per mouse; the number of times each mouse investigated the novel object; the time each mouse spent investigating the novel object; and the number of times the mouse entered the novel arm of the maze and the time spent in it.

\section{The investigation of learning and memory using the water task paradigm}

The water finding apparatus was designed to examine the ability of mice to gather information from a novel environment and to remember the location of a specific resource within that environment. The latency to relocate the resource (in this case, a water tube) after a period of deprivation was considered to be an indication of memory capacity of the mice.

The water task (Ettenberg et al., 1983) is constructed from plywood and consists of an open field with an alcove situated along the back wall of the enclosure. The interior of the open field is painted grey and the floor divided into 15 identical squares with fine black lines (about $2 \mathrm{~mm}$ ) to allow measurements of the animal's movements. A metal drinking tube, one of the same type used in the home cage, is inserted into the centre of the alcove ceiling with it's tip $5 \mathrm{~cm}$ (during the training session) or $7 \mathrm{~cm}$ (during the testing session) above the floor. A 60 watt lamp illuminated the apparatus.

During the training session, each mouse was placed in the right hand corner of the apparatus and its behaviour recorded over the next $3 \mathrm{~min}$. The following measurements were recorded: the number of times the animal crossed from one square to another in the open field; the number of times they touched or sniffed the water tube in the alcove (i.e. the number of approaches); and the number of rears performed per mouse. Animals that did not begin exploring within $3 \mathrm{~min}$ and those that did not find the water tube during the 3 min exploration period were not tested further, but the number of such animals were recorded.

After the training session, the mice were immediately returned to their home cage and deprived of water for $20 \mathrm{~h}$ before the test trial, which took place $48 \mathrm{~h}$ after the end of training. As for the training, testing took place over a $3 \mathrm{~min}$ period and the following recordings were taken: the number of seconds before they entered the alcove (i.e. entering latency); the number of seconds before they found the water tube on entering the alcove (i.e. finding latency); the number of seconds until they began to drink (i.e. drinking latency). Thus, the drinking latency consisted of the sum of the entering and finding latencies. In the case where a mouse did not drink or find the source of water during the test trial, it was automatically given a latency of $180 \mathrm{~s}$.

Investigation of anxiety in mice using the elevated plus maze The elevated plus maze (Pellow et al., 1985) is a novel conflict paradigm in which the drive to explore conflicts with the negative drive to avoid open or exposed areas.

The apparatus is made of wood and consists of two open arms and two enclosed arms with an open roof elevated $50 \mathrm{~cm}$ off the ground. The arms extend from a central platform. The enclosed arms are painted black while the central platform and two open arms are painted white.

Each mouse was placed on the central platform and allowed to explore the maze freely for a $3 \mathrm{~min}$ period. The following recordings were made for each mouse: the number of entries into the open arms and the closed arms, the time spent in the open arms and the closed arms; the time spent on the central platform; and the number of approach avoidances towards the open arm. An arm entry was defined as the entry of all four paws into one arm. At the end of testing, the number of entries into the open arms was expressed as a \% of the total number of arm entries. The time spent in the open arms was also expressed as a \% of the time spent on both the open and the closed arms.

\section{Larval counts}

The mice in each experiment were killed by cervical dislocation on the day following the last behavioural experiment. The brain was removed using a fine scissors and forceps. The Baermann procedure (Pritchard \& Kruse, 1982) was carried out in order to count the total number of larvae recovered from the whole brain of individual mice. The procedure for the examination of brain material is the same as that described in Cox \& Holland (1998).

It should be noted that the brain was the only organ examined for the presence of larvae in this study. This was because data from a previous investigation using the same strain of mice and a 100, 1000 and 3000 dose of $T$. canis ova revealed that by day 26 of infection larvae had accumulated in the brain and very few or no larvae were recorded from the liver, lung, kidney and muscle (Skerrett \& Holland, 1997).

\section{Statistical methods}

All statistical tests were carried out at the 95\% confidence limit. The data obtained for the ' $\mathrm{T}$ ' maze experiment, water task and elevated maze were analysed for inter-group differences using a one-way analysis of variance (ANOVA). Separate group comparisons were made using least significant difference (LSD) post hoc tests. In instances where the data was skewed, a log transformation was performed in order to normalize the data and those undergoing transformation are indicated in the text. 

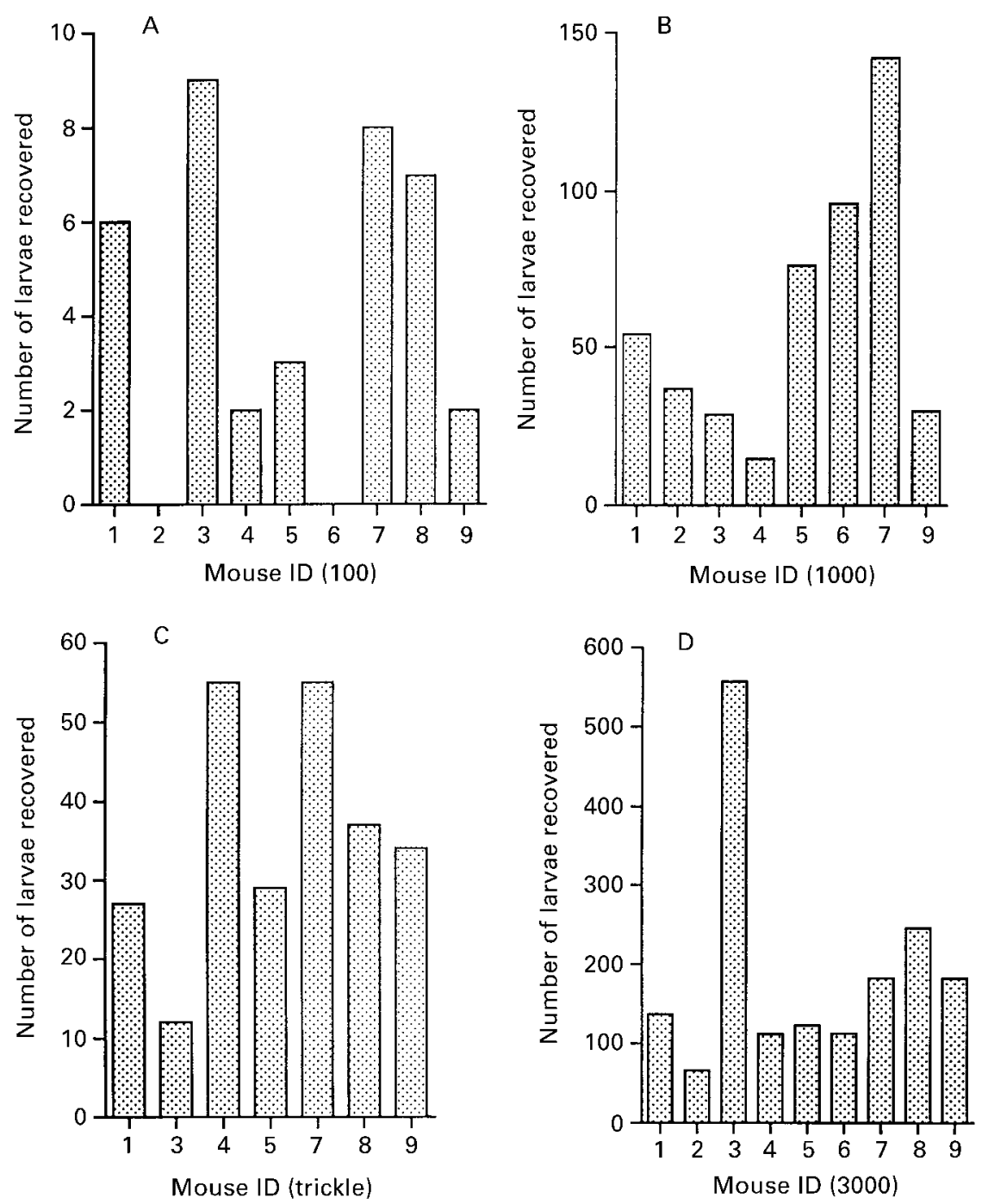

Fig. 1. Larval recoveries from the brains of individual mice infected with Toxocara canis. A, 100 ova dose; B, 1000 ova dose; C, trickle $(250 \times 4)$; D, 3000 ova dose. Changes in the y-axis scale should be noted.

\section{Results}

\section{Larval recovery}

The percentage larval recovery expressed in terms of the total dose of T. canis ova administered was highest in the 3000 group $(6.1 \%)$ and the 1000 group $(6.0 \%)$, followed by the $100(4.4 \%)$ and trickle (3.5\%) groups. The level of infection in the brain was lower in the mice receiving a multiple as opposed to an equivalent single dose of ova. The accumulation of T. canis in the brains of individual mice are displayed in fig. 1 . There was only a slight variation in the recoveries of larvae between the individual mice, which made up the 100 group (mean \pm SD $4.4 \pm 1.1$; range $0-9)$. Of the nine brains sampled, two were negative for $T$. canis larvae. The individual mice which made up the 1000 group showed more variation in the number of larvae they accumulated in the brain and were all positive for $T$. canis larvae (mean \pm SD $59.8 \pm$ 15.2; range 15-142). Investigation of the trickle dose showed that all individual mice were positive for larvae in the brain (mean \pm SD $35.5 \pm 5.5$; range $12-55$ ). The greatest variation was observed in the high dose group in which the mice were infected with 3000 ova (mean \pm SD $183.1 \pm 49.7$; range $66-557)$. All these brains were also positive for T. canis larvae.

\section{Division of infected mice into larval intensity groups}

On the basis of the number of larvae recovered from individual brains of mice, the infected mice were divided into three larval intensity groups. Mice which received an infective dose but contained no larvae (two from the 100 dose) were included with mice containing up to 15 larvae $(n=11$; mean \pm SD $5.8 \pm 4.9)$ and defined as low 
Table 1. The total number of arms entered and rears performed by mice on days of habituation and the difference in exploration from day 1 to day 2 .

\begin{tabular}{|c|c|c|c|c|c|c|}
\hline Behaviour & \multicolumn{4}{|c|}{ Infection } & $\mathrm{F}$ ratio & $P$ value \\
\hline Day 1 & $26.8 \pm 2.8$ & $31.4 \pm 1.8$ & $31 \pm 2.5$ & $21.1 \pm 2.6$ & 3.8 & 0.017 \\
\hline Day 2 & $24.2 \pm 2.7$ & $19.1 \pm 3.4$ & $20 \pm 2.5$ & $17.1 \pm 2.9$ & 1 & NS \\
\hline Difference & $2.6 \pm 4.3$ & $12.3 \pm 3.1$ & $10.8 \pm 1.6$ & $4.1 \pm 1.8$ & 3 & 0.046 \\
\hline Day 1 & $38.6 \pm 4.3$ & $46.2 \pm 3.8$ & $42.7 \pm 5$ & $35.4 \pm 4.5$ & 1.2 & NS \\
\hline Day 2 & $37.4 \pm 3.4$ & $24.8 \pm 5.3$ & $25.6 \pm 3.7$ & $23.2 \pm 3.9$ & 2.1 & NS \\
\hline Difference & $1.2 \pm 5.9$ & $21.4 \pm 4.9$ & $17.1 \pm 3.9$ & $12.1 \pm 4.2$ & 3.1 & 0.03 \\
\hline
\end{tabular}

Post hoc comparisons.

Arms entered: Day 1: low versus heavy, $P \leq 0.004$; moderate versus heavy, $P \leq 0.009$.

Difference between day 1 and 2: control versus low, $P \leq 0.02$; control versus moderate, $P \leq 0.05$; low versus heavy, $P \leq 0.03$.

Rears: Difference between day 1 and 2: control versus low, $P \leq 0.005$; control versus moderate, $P \leq 0.03$.

intensity infections. This included nine 100 dose mice and one 1000 dose and one trickle dose mouse respectively. Mice which contained 27 to 55 larvae were defined as moderate intensity infections $(n=10$; mean \pm SD $38.7 \pm 11.5$ ). This included four 1000 dose mice and six trickle dose mice. Mice which contained 66 to 557 larvae in the brain were defined as high intensity infections $(\mathrm{n}=12 ;$ mean $\pm \mathrm{SD} 169.3 \pm 131.9)$. This included nine 3000 dose mice and three 1000 dose mice.

\section{Exploratory behaviour in the ' $T$ ' maze on day one and two of habituation and the test day}

In general, the exploration of the arm entries and the start alley was higher for low and moderate intensity mice compared to the controls but lower in the high intensity mice. This was statistically significantly different for the number of start alley entries on day 1 (mean number $\pm \mathrm{SE}$ : control $6.2 \pm 0.6$; low $8.1 \pm 1$; moderate $7.5 \pm 0.6$; high $4.2 \pm 1)(\mathrm{F}$ ratio $=4 ; P \leq 0.0162, \mathrm{df}=$ 3,41 ; post hoc test low versus high $P \leq 0.003$; moderate versus high $P \leq 0.013$ ). This trend was also apparent for the number of rears on day 1 . On day 2, exploration of the start alley was reduced in all infected groups compared to the controls and differed significantly between the groups (control $7 \pm 1.2$; low $5 \pm 0.6$; moderate $5 \pm 0.5$; high $3 \pm 0.8)(\mathrm{F}$ ratio $=4, P \leq 0.0202 \mathrm{df}=$ 3,41 ; post hoc test control versus high $P \leq 0.002$ ) and this trend was also reflected in the arm entries and the entries and the number of rears. When the total number of arms entered was calculated (by pooling the left and right arm entries and the start alley entries) it can be seen that exploration in the ' $\mathrm{T}$ ' maze decreases in all groups of mice by day 2 of habituation (table 1). This decrease is much more pronounced for the low and moderately infected mice compared to the controls and when the difference between the two days is compared for the four groups it differs significantly (see table 1). A similar trend can be observed for the number of rears with a pronounced reduction occurring over the two day habituation period and a significant difference between all three infected groups compared to the control (table 1 ). There was no significant difference in the investigation of the maze by the different groups on the test day. However, exploration by the mice was shown to decrease over the three days as the familiarity of the environment increased.

Response to novelty of the LACA mice in the ' $T$ ' maze on the
test day

The trend for this experiment was that for all four measures relating to the novel object, the control and the low and moderate intensity mice were similar, but the high intensity group showed a divergence. For example, for the total time spent in the novel arm, the controls and the moderate intensity group spent approximately double the time compared to the high intensity group (mean seconds \pm SE : control 101.2 \pm 29 ; low $77.3 \pm 14.2$; moderate $100 \pm 26.1$; high $47.2 \pm 12.4$ ). The number of investigations of the novel object were higher for the control compared to all groups but markedly so for the high intensity group (control $8 \pm 1.3$; low $6 \pm 0.9$; moderate $6.2 \pm 1.4$; high $4.2 \pm 1.1$ ).

\section{Learning and memory in the water finding task paradigm}

\section{Training period}

There was a significant difference between the four groups for all three behaviours tested, i.e. the number of rears performed, the number of alcove entries and the number of squares crossed (log transformed data: $F$ ratio $=3.2, P \leq 0.0338$, df 3,$40 ; \mathrm{F}$ ratio $=3.2, P \leq 0.0352$ and $\mathrm{F}$ ratio $=4, P \leq 0.0149$ respectively). In general, infected mice performed less of these three behaviours compared to controls but no clear differences emerged between the three infected groups. Post hoc tests revealed that for all three behaviours each infected group differed significantly from the control.

\section{Testing period}

In general, the moderate and high intensity mice 
showed greater latency to enter the alcove, find the tube and to drink compared to the controls, although these differences did not reach statistical significance. This was particularly pronounced for the time it took the mice to find the drinking tube, with virtually double the time being taken by the moderate and high intensity mice compared to the low intensity group and the controls (mean seconds $\pm \mathrm{SE}$ : control $59 \pm 23.2$; low $40.4 \pm 9$; moderate $111.3 \pm 24.4$; high $94 \pm 22$ ). In addition, the control group contained the highest percentage of mice, $67 \%$, which actually drank once they found the water tube compared to the infected groups in which the proportion of mice that actually drank was much lower - low intensity $45.5 \%$, moderate intensity $20 \%$ and high intensity $25 \%$.

\section{Exploration and aversion in the elevated plus maze}

There were no statistically significant differences between the groups for any of the behaviours performed in the elevated maze but certain trends were apparent. The groups performed in a similar manner with regard to the entry to the enclosed arm of the maze and the time spent in it but for the open arm the moderate and high intensity mice spent more time compared to the controls (mean seconds $\pm \mathrm{SE}$ : control $8 \pm 5.1$; low $11.5 \pm 4.3$; moderate $21 \pm 11$; high $15 \pm 8.5$ ).

When the number of times the mice entered the open arm was expressed in terms of the total number of arm entries, these were higher for the infected mice and particularly so for the moderate intensity mice (control $5.2 \pm 3.1$; low $10 \pm 4$; moderate $19.5 \pm 3.5$; high $8 \pm 3.2$ ) $(\log$ transformed data: $\mathrm{F}$ ratio $=3.2 ; P \leq 0.0358 \mathrm{df}=$ 3,40 ) (post hoc tests control versus moderate $P \leq 0.03$; moderate versus low $P \leq 0.03$; moderate versus high $P \leq$ $0.01)$. When the time spent in the open arm was expressed in terms of the total time spent by each mouse, the trend was similar in that infected mice spent more time than the controls, and this was particularly pronounced for both the moderate and high intensity mice (control $5 \pm 3$; light $7.1 \pm 3$; moderate $12.4 \pm 6.2$; high $11 \pm 7$ ). Furthermore, the numbers of approaches/ avoidances was greater in the controls $(7.4 \pm 2.2)$ compared to the three infected groups (low $4.1 \pm 1.2$; moderate $4 \pm 1.1$; high $3 \pm 1$ ).

\section{Discussion}

Toxocara larval numbers recovered from the murine brain were low, as a percentage of dose, with the highest recovery from the 3000 dose and the lowest from the 100 and trickle doses. The distribution of T. canis larvae in the body is known to be dependent upon post-infection time, inoculum dose and the strain of mice used (El-Shazly et al., 1991; Dunsmore et al., 1983; Epe et al., 1994). In addition, the number of eggs used to initiate the infection in mice is an important criterion in determining the subsequent immune response mounted by the host against the parasite (Kayes et al., 1985). Previous work has shown that in this outbred LACA strain most larvae have left the other tissues and accumulated in the brain by day 26 post infection (Skerrett \& Holland, 1997). Clearly the level of brain infection was lower in mice who received a multiple rather than a single dose of 1000 ova and this is likely to be as a consequence of the immune response. This difference was also reflected in the lower standard deviation for the trickle group compared with the 1000 and 3000 dose groups.

The provision of four doses also resulted in mice with both lower and higher burdens compared to those derived from the 2000 dose given previously (Cox \& Holland, 1998). For example, the low intensity group described in this paper (which consisted of nine mice which received a 100 dose, one 1000 dose mouse and one trickle dose mouse) harboured an average larval burden of six larvae per brain (range 0-15) compared to values of 26.2 (range 7-45) and 40.2 (range 10-66) from the 2000 dose mice. This means that the behavioural response of mice with much lower burdens, which are likely to more realistically reflect the situation in wild rodents and infected humans, can be compared with moderate infections and considerably heavier infections (mean 169.3 (range 66-557) (nine 3000 dose and three 1000 dose mice) compared to averages of 96.5 (range 54-146) and 92.2 (range 68-129) in the 2000 dose mice. Dubinsky et al. (1995) examined the brains of 476 small mammals from Slovakia and found the numbers of larvae to range from one to 13 per brain with the peak average being $4.2 \pm 4.1$ in animals collected from a suburban location. The numbers of larvae harboured by the low intensity group described in this paper are very similar to those described by Dubinsky and colleagues.

Behavioural patterns were compared for the three infected groups of mice and controls and, in general, changes in behaviour were less pronounced in the low intensity group compared to the moderate and high intensity groups. Despite this, this group did show some divergence from the control mice.

Exploration is an essential but risky behaviour carried out by small rodents. One of the adaptive values of successful exploration lies in an enhanced ability to escape from predators (Montgomery \& Gurnell, 1985). The exploratory behaviour of the mice in the ' $\mathrm{T}$ ' maze differed between the groups, with low and moderate intensity mice being more exploratory compared to the controls and high intensity mice showing less exploration. Over the two days of habituation, exploration was reduced in all groups as the mice became more familiar with the environment but this reduction was more pronounced for the low and moderate intensity groups compared to the high intensity group. This implies that normal exploration is impaired in the high intensity group of mice and this could influence predation and hence enhanced transmission of the parasite in the wild. In addition, the high intensity group spent less time in the novel arm and made less investigations of the novel object compared to the control and the two other infected groups. It is likely that these reductions in exploration and response to novelty result from the non-specific debilitatory effect of heavy infection rather than enhanced neophobia.

Burright et al. (1982) infected mice with graded doses of $T$. canis and showed that they differed in their response to a familiar and novel environment. Mice infected with a dose of 1000 larvae never entered the novel environment whereas mice which received a lower dose of 5000 and 250 ova did; however, control mice entered the novel 
environment on more occasions and spent significantly more time than any of the infected groups. Dolinsky et al. (1981) also showed that infected outbred mice, which received a single dose of 1000 ova, showed a longer latency to enter a novel cage compared to control mice. In contrast, Hay \& Aitken (1984) found outbred mice, infected with 1000 ova, to be less cautious and to display greater preference for a blocked novel arm in a $\mathrm{Y}$ maze compared to control mice.

Changes in exploration have also been observed for the protozoan parasite Toxoplasma gondii that, like Toxocara canis, has a predilection for the brain in its intermediate or abnormal hosts. Laboratory mice have been shown to be less responsive to a novel arm in a ' $Y$ ' maze although their activity was greater during habituation and test trials (Hutchison et al., 1980; Hay et al., 1983, 1984). In contrast, Toxoplasma gondii-infected wild and hybrid rats also displayed increases in activity but were significantly more responsive to novel stimuli than control rats, indicating marked reductions in normal neophobic behaviour associated with rats (Webster, 1994; Webster et al., 1994; Berdoy et al., 1995).

In terms of learning and memory, infected mice showed a greater latency to enter the alcove, find the water tube and to drink from it compared to controls and this was more pronounced for the moderate and high intensity groups suggesting possible memory impairment. During the training period, all the infected mice showed reduced exploration compared to the controls and this could affect the ability of the animal to remember the location of the tube or result in the failure of the mouse to identify this resource on the training day. It should also be noted that the drive to enter the alcove may be driven more by a desire to locate the alcove itself, as a consequence of the murine preference for narrow, dark places, rather than a need to locate the water source.

Toxocara canis infection in laboratory rats (Olson \& Rose, 1966) and laboratory mice (Dolinsky et al., 1981) has been shown to affect learning. Dolinsky and colleagues found that infected mice performed less well on a stepthrough passive avoidance task with the criterion to avoid shock taking longer in infected mice compared to controls. Olson \& Rose (1966) using very high dose of $T$. canis ova showed that infected rats displayed decreased ability to learn simple and complex maze problems but that the parasite did not affect memory as measured by the rats performance before the infection was administered and after. Rats infected with 5000 ova did not show significant differences from controls, but as the dosage increased to 20,000 ova the rats averaged significantly more errors than the controls during the second week of infection. Larval recoveries from the brain were low, e.g. only $1 \%$ for rats infected with $5000-7500$ ova which increased to $4 \%$ in rats infected with 20,000 ova.

Poor spatial learning has been demonstrated in mice with light and moderate infections of the intestinal nematode Heligimosomoides polygyrus (Kavaliers \& Colwell, 1995) and it has been suggested that the poorer performance shown by infected mice could result in an inability to gain the same information from environmental cues as the control mice. Stretch et al. (1960) studied the ability of Schistosoma mansoni-infected mice to discriminate between black and white in a water maze discrimination task and found a learning deficit in infected mice. They also suggested that, given that there was a reduction in the swimming levels of the infected mice, this may have accounted for the impairments in learning and in later experiments they concluded that a motivational change rather than a change in learning ability caused infected mice to display poorer performances. The reduction in the ability of an infected animal to familiarize itself with its immediate environment, whether due to a reduction in learning and memory or motivation, may also increase the risks associated with predation.

Moderate and high intensity mice entered the open arm of the elevated maze more frequently and spent more time in it, particularly if this was expressed in terms of the total number of arm entries and the total time spent. This implied that more intensely infected mice were less aversive than control mice and this reduction in anxiety coupled with lack of inhibition to open areas could increase the risk of being located by a predator. This study incorporated two elements which are infrequently used in the elevated maze test - time spent on the central platform, which may be more aversive than the areas protected by walls and the number of approaches/avoidances which is a good measure of the cautiousness of the animal. No differences were observed between the four groups for time spent on the central platform but the controls clearly showed more approaches/avoidances compared to the infected groups.

Rodgers \& Johnson (1995) undertook a factor analysis to examine the relationship between standard spatiotemporal measures in the elevated plus maze and a range of specific defensive behaviours in mice. Factors relating not only to anxiety but also locomotory activity, decision making, risk assessment, vertical activity and exploratory behaviour were identified. In addition, these authors commented on how different behavioural tests tap into different facets of anxiety. In our previous study, where anxiety was measured by means of the light/dark box and the response to predator odour, mice with low infection in the brain (70 or less larvae) which are roughly equivalent to the moderate infection in these experiments, displayed a greater level of risk behaviour than control or high intensity mice (Cox \& Holland, 1998). This indicates how intensity of infection, in addition to the type of behavioural test employed, may influence observed changes in behaviour.

The conclusions that can be drawn from the present investigation are, firstly, that brain burden of $T$. canis larvae increases with the dose of ova administered and there is significant variation at each individual dose which supports our previous finding with a single dose of 2000 ova. Secondly, in general, the results for the low intensity group of mice indicate that larval burdens of that size have less marked effects on murine exploration, memory and anxiety. Thirdly, in contrast, more pronounced effects were observed in the moderate and high intensity infected mice - for exploration and response to novelty, the effects were more marked in the high intensity group whereas for learning, memory and anxiety effects could be observed in both groups. This study did not provide any measures of the pathological 
impact of the parasite and clearly the side effects of infection are more likely to operate at higher infection levels which in turn may affect several different behaviours. In humans, Taylor et al. (1988) described abdominal pain, nausea, limb pains and lethargy among a large group of other non-specific symptoms associated with raised titres to Toxocara ( $>0.3$ optical density). Under natural conditions, changes in behaviour regardless of their cause may predispose the paratenic host to predation.

Finally, these results suggest that a 3000 egg dose may be an inappropriate level of infection to administer to mice used in behavioural testing. Dose levels of up to 2000 are sufficient to detect subtle differences in behaviour (Cox \& Holland, 1998) and investigations involving memory and anxiety impairments may be investigated successfully without interfering with the motivational level of the mice which appears to be affected at higher doses.

\section{Acknowledgements}

The authors are very grateful to Peter Stafford for his excellent technical assistance. This research was partly funded by the Irish American Partnership.

\section{References}

Beaver, P.C., Synder, C.H., Carerra, G.M., Dent, J.H. \& Lafferty, J.W. (1952) Chronic eosinophilia due to visceral larva migrans. Pediatrics 9, 7.

Berdoy, M., Webster, J.P. \& Macdonald, D.W. (1995) Parasite-altered behaviour: is the effect of Toxoplasma gondii specific? Parasitology 111, 403-409.

Burren, C.H. (1971) The distribution of Toxocara canis larvae in the central nervous system of the mouse. Transactions of the Royal Society of Tropical Medicine and Hygiene 65, 450-453.

Burright, R.G., Donovick, P.J., Dolinsky, Z.Z., Hurd, Y. \& Cypess, R. (1982) Behavioural changes in mice infected with Toxocara canis. Journal of Toxicology and Environmental Health 10, 621-626.

Cox, D.M. (1996) The effect of Toxocara canis infection on the paratenic host behaviour of two strains of mice. Unpublished PhD thesis, Trinity College Dublin, Ireland.

Cox, D.M. \& Holland, C.V. (1998) The relationship between numbers of larvae recovered from the brain of Toxocara canis infected mice and social behaviour and anxiety in the host. Parasitology 116, 579-594.

Dolinsky, Z.S., Burright, R.G., Donovick, P.J., Glickman, L.T., Babish, J., Summers, B. \& Cypess, R.H. (1981) Behavioural effects of lead and Toxocara canis in mice. Science 213, 1142-1144.

Dubinsky, P., Havasiova-Reiterova, K., Petko, B., Hovorka, I. \& Tomasovicova, O. (1995) Role of small mammals in the epidemiology of toxocariasis. Parasitology 110, 187-193.

Dunsmore, J.D., Thompson, R.C.A. \& Bates, I.A. (1983) The accumulation of Toxocara canis larvae in the brains of mice. International Journal for Parasitology 13, 517521.
El-Shazly, A.M., Handousa, A.E., Youssef, M.E. \& AboShady, A.F. (1991) The effect of the initial inoculum dose and post-infection period on larval dispersal in experimental murine toxocariasis. Journal of the Egyptian Society of Parasitology 21, 785-789.

Epe, C., Sabel, T., Schneider, T. \& Stoye, M. (1994) The behaviour and pathogenicity of Toxocara canis larvae in mice of different strains. Parasitology Research 80, 691695.

Ettenberg, A., Le Moal, M., Koob, G.F. \& Bloom, F.E. (1983) Vasopressin potentiation in the performance of a learned appetitive task: reversal by a pressor antagonist analog of vasopressin. Pharmacology, Biochemistry and Behaviour 18, 645-671.

Hay, J. \& Aitken, P.P. (1984) Experimental toxocariasis in mice and their effect on behaviour. Annals of Tropical Medicine and Parasitology 78, 145-155.

Hay, J., Hutchison, W.M., Aitken, P.P. \& Graham, D.I. (1983) The effect of congenital and adult-acquired Toxoplasma infections on activity and responsiveness to novel stimulation in mice. Annals of Tropical Medicine and Parasitology 77, 483-495.

Hay, J., Aitken, P.P. \& Graham, D.I. (1984) Toxoplasma infection and response to novelty in mice. Zeitschrif für Parasitenkunde 70, 575-588.

Hutchison, W.M., Aitken, P.P. \& Wells, W.P. (1980) Chronic Toxoplasma infections and familiarity novel discrimination in the mouse. Annals of Tropical Medicine and Parasitology 74, 145-150.

Holland, C.V. (1997) Epidemiology of toxocariasis in Ireland: human, animal and environmental aspects. pp. 52-64 in Holland, C.V. (Ed.) Modern perspectives on zoonoses. Dublin, Royal Irish Academy.

Holland, C.V., O'Lorcain, P., Taylor, M.R.H. \& Kelly, A. (1995) Sero-epidemiology of toxocariasis in school children. Parasitology 110, 535-545.

Kavaliers, M. \& Colwell, D.D. (1995) Reduced spatial learning in mice infected with the nematode Heligmosomoides polygyrus. Parasitology 110, 591-597.

Kayes, S.G., Omholt, P.E. \& Grieve, R.B. (1985) Immune responses of $\mathrm{CBA} / \mathrm{J}$ mice to graded infection with Toxocara canis. Infection and Immunity 48, 697-703.

Montgomery, W.I. \& Gurnell, J. (1985) The behaviour of Apodemus. pp. 89-108 in Flowerdew, J.R., Gurnell, J. \& Gipps, J.H.W. (Eds.) The ecology of woodland rodents, bank voles and wood mice. Oxford, Oxford Science Publications.

Moore, J. \& Gotelli, N.J. (1990) A phylogenetic perspective on the evolution of altered host behaviours: a critical look at the manipulation hypothesis. pp. 193229 in Barnard, C.J. \& Behnke, J.M. (Eds.) Parasitism and host behaviour. London, Taylor and Francis.

Olson, L.J. \& Rose, J.E. (1966) Effect of Toxocara canis infection on the ability of white rats to solve maze problems. Experimental Parasitology 19, 77-84.

Pellow, S., Chopin, P., File, S.E. \& Briley, M. (1985) Validation of open:closed arm entries in an elevated plus-maze as a measure of anxiety in the rat. Journal of Neuroscience Methods 14, 149-167.

Pritchard, M. \& Kruse, G.O.W. (1982) The collection and preservation of animal parasites. $13 \mathrm{pp}$. Lincoln and London, University of Nebraska Press.

Rodgers, R.J. \& Johnson, N.J.T. (1995) Factor analysis of 
spatiotemporal and ethological measures in the murine elevated plus-maze test of anxiety. Pharmacology Biochemistry and Behavior 52, 297-303.

Shields, J.A. (1984) Ocular toxocariasis. A review. Opthalmology 28, 361-381.

Skerrett, H. \& Holland, C.V. (1997) Variation in the larval recovery of Toxocara canis from the murine brain: implications for behavioural studies. Journal of $\mathrm{Hel}$ minthology 71, 253-255.

Sprent, J.F.A. (1955) On the invasion of the central nervous system in nematodes II. Invasion of the nervous system in ascariasis. Parasitology 45, 41-58.

Stretch, R.G.A., Leytham, G.W.H. \& Kershaw, W.E. (1960) The effect of acute schistosomiasis upon learning in rats under different levels of motivation. Journal of Tropical Medicine and Parasitology 54, 487-492.

Summers, B., Cypess, R.H., Dolinsky, Z.S., Burright,
R.G. \& Donovick, P.J. (1983) Neuropathological studies of experimental toxocariasis in lead exposed mice. Brain Research Bulletin 10, 547-550.

Taylor, M.R.H., Keane, C.T., O'Connor, P., Mulvihill, E. \& Holland, C.V. (1988) The expanded spectrum of toxocaral disease. Lancet i, 692-694.

Webster, J.P. (1994) The effect of Toxoplasma gondii and other parasites on activity levels in wild and hybrid Rattus norvegicus. Parasitology 109, 583-589.

Webster, J.P., Brunton, C.F.A. \& Macdonald, D.W. (1994) Effect of Toxoplasma gondii upon neophobic behaviour in wild brown rats, Rattus norvegicus. Parasitology 109, $37-43$. 